\title{
GA²LEN-Studien: wichtige Infos für die Allergiediagnostik
}

\author{
Der Hautpricktest gehört zur Standarddiagnostik der allergologischen Praxis. In einer \\ paneuropäischen Hautpricktest-Studie des GA²LEN-Netzwerks wurde nun eine vereinheitlichte \\ Standardtestbatterie, die Verteilung von Sensibilisierungen im europäischen Raum und die \\ klinische Relevanz positiver Hauttest-Befunde untersucht.
}

Ein Projekt des Global Allergy and Asthma European Network (GA²LEN) ist die europaweite Standardisierung von Hautprick-Testungen für die Diagnostik einer IgE-vermittelten Sensibilisierung gegen Inhalationsallergene. Gleichzeitig wurde geklärt, ob die bisher übliche Testung mit den landestypischen "lokalen“" Inhalationsallergenen tatsächlich alle relevanten Sensibilisierungen aufdeckt.

Im Rahmen der paneuropäischen Pricktest-Studie wurde zunächst eine Testbatterie mit den 18 häufigsten Inhalationsallergenen in Europa zusammengestellt. Die Allergene und die Testdosierungen wurden auf Basis von epidemiologischen Daten zu Sensibilisierungsrate, Kreuzreaktivität und klimatischen Faktoren ausgewählt. Die Testextrakte stellten drei führende Allergenhersteller zur Verfügung. In $17 \mathrm{GA}^{2} \mathrm{LEN}-Z$ entren in 14 europäischen Ländern wurden anschließend die Ergebnisse der standardisiert durchgeführten Pricktests von 3.034 Personen (Durchschnittsalter 33 Jahre) gesammelt.

Erwartungsgemäß ergaben sich landestypische Allergiemuster, aber auch unerwartete Sensibilisierungen, die unter anderem auf die hohe Mobilität der Bevölkerung zurück geführt werden können. Die Sensibilisierung gegen Innen- raumallergene wie Katzen- und Hundeallergene war in den nördlichen Ländern vergleichsweise hoch (30-56\%). Gegen Hausstaubmilben waren vor allem Bewohner der nördlichen Länder sowie der Mittelmeerregionen allergisch (bis zu $68 \%$ ), Deutschland lag mit 12\% im Mittelfeld. Bei den „Außen-Allergenen“ dominierten Frühblüherallergien in Nordund Mitteleuropa (bis zu 57\%), allerdings waren solche Sensibilisierungen auch noch in Mittelmeerländern wie Italien (ca. 10\%) relevant. Bäume und Sträucher des Südens wie Zypressen verursachten auch in den südlichen Ländern nicht sehr häufig Allergien, Hauptallergen war hier die Olive mit einer Sensibilisierungsrate von beispielsweise $23 \%$ in Italien. Aufgrund der Kreuzreaktivität Olive-Esche und der hohen Mobilität lag die Zahl positiver Hautreaktionen gegen Olive aber auch in nördlicheren Ländern noch recht hoch, in Deutschland betrug sie knapp $10 \%$. Gräserallergien sind ein paneuropäisches Problem, Sensibilisierungen gegen Kräuter waren unter den Patienten in Ungarn und Polen relativ häufig.

Ziel der paneuropäische Pricktest-Studie war zudem die Evaluation der klinischen Relevanz von positiven HauttestBefunden. Hierbei waren im Allergen-
gruppen-Mittel über $60 \%$ der nachgewiesenen Sensibilisierungen klinisch relevant. Je mehr Einzeltests bei einem Patienten positiv ausfielen, desto höher war die Wahrscheinlichkeit für eine allergische Rhinitis und/oder allergisches Asthma und atopisches Ekzem - ein Befund, der das Konzept einer atopischen Veranlagung unterstützt. Die klinische Relevanz der gefundenen positiven Hauttests hing vom getesteten Allergen und vom jeweiligen Untersuchungsland ab. Die Ursachensuche für diesen Befund steht noch aus.

Fazit: Allergie-Hauttests sollten europaweit mit einer Standardtestreihe der 18 häufigsten Inhalationsallergene durchgeführt werden. Durch die Vereinheitlichung sind die Ergebnisse vergleichbar. Die klinische Relevanz positiver Hauttests liegt bei über $60 \%$.

bk

Heinzerling $L M$ et al. GA²LEN skin test study I: GA²LEN harmonization of skin prick testing: novel sensitization patterns for inhalant allergens in Europe. Allergy 2009; 64: 1498-1506.

Burbach GJ et al. GA²LEN skin test study II: clinical relevance of inhalant allergen sensitizations in Europe. Allergy 1009; 64: 1507-15 